\title{
Health Technology Assessment of Ultraviolate light based imaging technology to reduce burden of hospital acquired infections: A Study Protocol
}

Komal Shah ( $\nabla$ kshah@iiphg.org )

Indian Institute of Public Health Gandhinagar

Priya Kotwani

Indian Institute of Public Health Gandhinagar

Somen Saha

Indian Institute of Public Health Gandhinagar

\section{Protocol}

Keywords: UV light based imaging technology, Health Technology Assessment, Hospital Acquired Infections, Cost-effectiveness

Posted Date: June 1st, 2020

DOI: https://doi.org/10.21203/rs.3.rs-21568/v2

License: (c) (i) This work is licensed under a Creative Commons Attribution 4.0 International License. Read Full License 


\section{Abstract}

Background: HAls impart a huge clinical and economic burden in India. Healthcare worker's compliance to hand hygiene protocol can play an important role in preventing the transmission of cross-infection and thus reducing the HAls. Over last few years, there has been a wide use of ultraviolate (UV) based fluorescent markers for assessing quality of hand hygiene. This Health Technology Assessment (HTA) study will be conducted with the aim to identify low cost, easy and feasible strategy having potential to get integrated in the current health system to deal with issue of HAI in ICU settings.

Methods: The study will be conducted in three phases. The impact of innovation on hand hygiene compliance will be assessed quantitatively by undertaking meta-analysis of secondary literature on UV based imaging tool in improving hand hygiene compliance in ICUs. Following this, the feasibility of using this innovation in developing country like India will be assessed. Decision Analytic modelling will be conducted for cost-effectiveness analysis using health systems perspective. Cost per ICU stay (day) and QALY gained will be calculated and ICER will be reported to comment on the cost-effectiveness of the innovation.

Discussion: The HTA study will provide a comprehensive overview of the effectiveness of UV light based imaging device in reduction of HAls. The results of this HTA study will generate evidence for the decision makers for its incorporation in public healthcare system of India.

Systematic review registration: The protocol has been registered in Prospero (Prospero Id: CRD42018108960)

\section{Background}

Studies across the globe showed that hospital-acquired infections (HAls) also known as nosocomial infections impart colossal economic burden on health care system due to prolongation of hospital stay, and increased morbidity and mortality [1]. As the name suggests, hospital settings are at a highest risk of acquiring these infections especially the intensive care units (ICUs) and operation theatres. ICU are reported to house several life-threatening bacteria such as Acinetobacter, Pseudomonas, and Klebsiella and are often associated with spread of diseases such as pneumonia, bloodstream infection and urinary tract infection. The major contributors of these infections are lack of adherence to infection control protocols, vigilant surveillance system, use of outdated technology and tendency to overuse antibiotics. Constant efforts are being directed towards reducing its incidence, still HAl remains a persistent problem not only for the low and middle income countries but also for the developed world. It is also known that implementation of proper guidelines and preventing the infection with bacterial resistance rather than treating it with antibiotics will help reduce the disease burden.

As learnt from lessons of Semmelweis, hand hygiene has proven efficacy in directly reducing the HAls and is also a cost effective measure due to improvement in patient safety. Healthcare worker's compliance to hand hygiene protocol can play an important role in preventing the transmission of cross- 
infection and thus reducing the HAls. Involuntary contamination of the hands of healthcare workers contribute to $20-40 \%$ of the HAl with alarmingly high incidence of HAl in India (11-83\%). Unfortunately, compliance to this simple strategy of hand hygiene for HAI preventions is found to be very low and is difficult to improve, especially in low and middle income countries (LMICs) where work pressure and patient to health care provider ratio are inherent challenges. One report from India showed that even in critical care units the compliance to hand hygiene amongst health care workers remains to be mere $37 \%$, which may ultimately lead to fatal consequences [2]. Apart from this, HAl is also associated with antimicrobial resistance (AMR) as the approach taken at times is to prevent hospital acquired infections with antibiotics which leads to many people consuming large proportion of antibiotics which leads to resistance towards those drugs.

Over last few years, there has been a wide use of ultraviolate (UV) based fluorescent markers for assessing quality of hand hygiene [3-22]. This technological intervention involves performance of recommended WHO six steps using Alcohol-based hand rub with a fluorescent marker by health care workers followed by viewing under UV based lamp to identify the quality of hand hygiene. This exercise discriminates contaminated areas of hands through fluorescence where the areas with proper distribution of Alcohol-based hand rub are seen white under the UV lamp while the unrubbed areas or the contaminated ones appear dark. The use of this innovative device was shown to be effective in improving hand hygiene compliance amongst the healthcare workers. The brief description of the technology is provided in figure 1.

HAls impart a huge clinical and economic burden in India. With this enormous clinical and economic burden of HAl in India and its association with hand-hygiene compliance, critical evaluation of the available strategies reporting its effectiveness in improving hand hygiene compliance of health care workers is need of an hour. This Health Technology Assessment (HTA) study will help us to identify low cost, easy and feasible strategy having potential to get integrated in the current health system to deal with issue of HAI in ICU settings. The objectives of the study are

- To undertake evidence synthesis through meta-analysis and assess efficacy of an Innovative tool involving UV light based digital imaging for improving hand hygiene compliance in ICU of hospitals across the globe

- Using a decision tree model approach, to evaluate cost-effectiveness of the innovation for reducing HAl through improvement in hand hygiene compliance in Indian context.

\section{Methods}

The principle aim of this HTA study is to assess feasibility of the UV based imaging innovation on both clinical and cost aspects. However, as the technology is relatively new for Indian context, hence we aim to evaluate the clinical efficacy of the innovation using global evidences. For cost-effectiveness of the innovation, $\mathrm{HAl}$ will be used as a surrogate marker of the impact associated with the implementation of the innovation. For the same, costing data will be retrieved from Indian studies. 
The study will be conducted in three phases. In the first phase, the impact of innovation on hand hygiene compliance will be assessed through undertaking a systematic review and meta-analysis approach on effect of UV based imaging tool in improving hand hygiene compliance in ICUs. While in the phase to the translation of clinical benefits of hand hygiene in terms of reduced incidence of HAI in Indian context will be measured. The phase 3 comprises of cost-effectiveness analysis of the innovation using decision analytic modelling.

The overview of the methodology is shown in figure 2.

The methodology that will be followed to undertake the systematic review and meta-analysis is described below

We will undertake evidence synthesis using quantitative methods (meta-analysis) assessing potential of UV light based imaging technique in inducing behavioral changes in health care workers of ICU across the globe. The impact will be evaluated in terms of improvement in hand hygiene compliance rate. The overall effect will be expressed in terms of pooled odds ratio.

\section{Criteria for considering studies for this review:}

Types of studies: The articles incorporated were all kind of studies including cross-sectional, case control, randomized controlled trials (RCTs), review and meta-analysis with no restriction to language and calendar date. Reference articles of the recovered articles were also screened for potential studies.

Types of participants: Health care workers of ICU

Types of interventions: UV light based imaging technology to assess hand hygiene compliance

Types of outcome measures: Hand hygiene compliance rate

\section{Search methods for identification of studies}

We will use criteria and standard methods of Cochrane and Cochrane infectious diseases.

\section{Electronic searches}

For assessing impact of UV light based innovative technique on hand-hygiene: For retrieving articles reporting impact of UV light based fluorescent technique on hand hygiene compliance, we will be searching five databases as per following search strategy and study selection criteria: 1] PUBMED (until September 2019) 2] EMBASE (1980 to September 2019) 3] CINAHL (until September 2019) 4] Cochrane 
CENTRAL (until September 2019) 5] Clinical trials registries for ongoing and recently completed trials (clinicaltrials.gov; World Health Organization International Trials Registry). These databases were explored independently by the authors. The search terms to be used are: "Hand washing" OR "Hygiene" OR "Hand rubbing" OR "Compliance" AND "Ultraviolate" OR "UV" OR "Ultra-violate" OR "Fluorescent".

Two authors will perform the final selection of studies for inclusion and exclusion by assessing full text and extracting the data independently. The third author will perform cross-verification of the extracted data. Differences of opinions will be resolved through discussion and mutual consensus.

\section{Searching other resources}

We will seek additional citations by using references in articles retrieved through searches. We will contact subject experts to identify unpublished and ongoing studies specially for retrieval of the costing data. We will contact authors of published trials to clarify or provide additional information if criteria for methodological assessment are not explicit in their publication. In cases of cluster-randomized trials, we will contact study authors for an estimate of design effect if needed. Two review authors will independently screen candidate articles to check eligibility for inclusion in the review.

\section{Selection of studies}

Two review authors will independently screen the titles and abstracts of articles identified by searches for eligibility. We will classify these studies as included, unclear, or excluded. We will retrieve full articles for studies that do not provide an abstract or that have a limited abstract and will assess them for inclusion. Two review authors will independently assess for inclusion full articles classified as 'include' or 'unclear' using a standardized form with explicit inclusion and exclusion criteria. These two review authors will resolve disagreements by discussion and, if required, by consultation with a third review author.

\section{Data extraction and management}

Two review authors will independently extract data from each included study using a predesigned data extraction form. We will try to contact trial authors to request incompletely reported data. We will extract the following information: general information (study ID, date of extraction, title, authors, and source of study if not published); study characteristics (study design, participants, and inclusion/exclusion criteria used in the study); details of interventions; and details necessary for 'Risk of bias' assessment. We will resolve disagreements between review authors by discussion or by consultation with a third review author. 


\section{Assessment of risk of bias in included studies}

We will use the 'Risk of bias' assessment tool and criteria set out in the Cochrane Handbook for Systematic Reviews of Interventions to assess risk of bias for included studies [23]. Two review authors will independently assess risk of bias in the included studies by assessing randomization sequence generation; allocation concealment; blinding of participants, personnel, and outcome assessors; incomplete outcome data; selective outcome reporting; and other sources of bias. We will resolve disagreements through discussion or by consultation with a third review author. We will evaluate and report the following results in 'Risk of bias' tables.

- Selection bias (random sequence generation and allocation concealment): For each included study, we will categorize risk of selection bias as:

- low risk - adequate (any truly random process, e.g. random number table; computer random number generator; coin tossing; shuffling of cards; throwing of dice; drawing of lots; minimization);

- high risk - inadequate (any non-random process, e.g. odd or even date of birth; hospital or clinic record number); or

- unclear risk - insufficient information about the sequence generation process to permit judgement of 'low risk' or 'high risk'.

- Allocation concealment: For each included study, we will categorize risk of bias regarding allocation concealment as:

- low risk - adequate (e.g. telephone or central randomization; consecutively numbered, sealed opaque envelopes);

- high risk - inadequate (open random allocation; unsealed or non-opaque envelope alternation; date of birth); or

- unclear risk - no or unclear information provided.

- Performance bias: For each included study, we will categorize methods used to blind study personnel from knowledge of which intervention a participant received. As our study population will consist of neonates, all will be blinded to the study intervention. We will categorize risk of performance bias as:

- low risk - adequate for personnel; 
- high risk - inadequate for personnel (aware of group assignment); or

- unclear risk - no or unclear information provided.

- Detection bias: For each included study, we will categorize methods used to blind outcome assessors from knowledge of which intervention a participant received. We will categorize methods used with regards to detection bias as:

- low risk - adequate (follow-up was performed with assessors blinded to group assignment);

- high risk - inadequate (assessors at follow-up were aware of group assignment); or

- unclear risk - no or unclear information provided.

- Attrition bias: For each included study and for each outcome, we will describe completeness of data including attrition and exclusions from the analysis. We will note whether attrition and exclusions were reported, numbers included in the analysis at each stage (compared with total randomized participants), reasons for attrition or exclusion when reported, and whether missing data were balanced across groups or were related to outcomes. When trial authors reported or supplied sufficient information, we will re-include missing data in the analyses. We will categorize methods with respect to risk of attrition bias as:

- low risk - adequate (< $10 \%$ missing data);

- high risk - inadequate (> 10\% missing data); or

- unclear risk - no or unclear information provided.

- Reporting bias: For each included study, we will describe how we investigated risk of selective outcome reporting bias and what we found. We will assess methods as:

- low risk - adequate (when it is clear that all of the study's pre-specified outcomes and all expected outcomes of interest to the review have been reported);

- high risk - inadequate (when not all of the study's pre-specified outcomes have been reported; when $\geq 1$ reported primary outcomes were not pre-specified; when outcomes of interest are reported incompletely and so cannot be used; when study fails to include results of a key outcome that will be expected to have been reported); or 
- unclear risk - no or unclear information provided (study protocol was not available).

- Other bias: For each included study, we will describe any important concerns that we had about other possible sources of bias (e.g. whether a potential source of bias was related to the specific study design, whether the trial was stopped early owing to some data-dependent process). We will assess whether each study was free of other problems that could put it at risk of bias, as:

- low risk - no concerns of other bias raised;

- high risk - concerns raised about multiple looks at data, with results made known to investigators, difference in numbers of participants enrolled in abstract and in final publications of the paper; or - unclear risk - concerns raised about potential sources of bias that could not be verified by contacting trial authors. We plan to explore the impact of level of bias by undertaking sensitivity analyses.

\section{Measurement of treatment effect}

We will perform statistical analysis according to statistical guidelines referenced in the Cochrane Handbook for Systematic Reviews of Interventions [23] For dichotomous outcomes, we will express measures of effects as typical odds ratios (ORs) and typical odds differences (ODs) with $95 \%$ confidence intervals (Cls). If the RD is statistically significant, we will calculate the number needed to treat for an additional beneficial or harmful outcome. For continuous outcomes, we will express measures of effect as weighted mean differences (MDs) with $95 \%$ Cls. In the event that continuous data are reported on different continuous scales, we will standardize outcomes, when possible, to calculate the standardized Mean Difference.

\section{Unit of analysis issues}

Analyses will consider the level at which randomization was done - individual or cluster. In the event that cluster-randomized studies are included, we will appropriately adjust for clustering; and we will multiply the standard error derived from the confidence interval of the effect estimate by the square root of the design effect. We will use the generic inverse variance method in Review Manager 5 to perform metaanalysis using inflated variances [24].

\section{Dealing with missing data}


We will contact trial authors to request missing data and will use imputation methods when necessary. We will clearly label analyses by including imputed data.

\section{Assessment of heterogeneity}

When data from included trials can be pooled, we will assess statistical heterogeneity via visual inspection of forest plots of included trials, using the $\mathrm{Chi}^{2}$ test and the $\mathrm{I}^{2}$ statistic. We will examine trial characteristics (participants, design, interventions, outcomes, and risk of bias) to identify the source of any observed heterogeneity. We would use cut-offs recommended by Cochrane Infectious diseases for results of the $\mathrm{I}^{2}$ test: $<25 \%$ none, $25 \%$ to $49 \%$ low, $50 \%$ to $74 \%$ moderate, and $75 \%+$ high heterogeneity.

\section{Assessment of reporting biases}

We will assess reporting biases by trying to identify whether the study was included in a trial registry, whether a protocol is available, and whether the methods section provides a list of outcomes. We will compare the list of outcomes from those sources versus outcomes reported in the published paper. We will create an inverted funnel plot to check for possible publication bias if a sufficient number of studies are available for specific outcomes.

\section{Data synthesis}

If studies are found to be similar, we will use Review Manager 5 software to combine in the meta-analysis data for outcomes from studies that meet the inclusion criteria [24]. We will

perform statistical analyses according to statistical guidelines of the Cochrane Handbook for Systematic Reviews of Interventions and Cochrane Infectious Diseases [23]. We will conduct a fixed-effect metaanalysis if appropriate. We will not conduct meta-analysis when a high level of heterogeneity is evident. We will provide only a narrative summary of trial findings if data cannot be combined in a meta-analysis. In the event that continuous data are reported on different continuous scales, we will standardize outcomes, when possible, to calculate the standardized MD.

\section{Quality of evidences}

We will use the GRADE approach, as outlined in the GRADE Handbook, to assess the quality of evidence for the following (clinically relevant) outcomes: percent improvement in hand hygiene compliance, incidence of HAl [25]. Two review authors will independently assess the quality of evidence for each of 
the outcomes above. We will consider evidence from RCTs as high quality but will downgrade the evidence one level for serious (or two levels for very serious) limitations on the basis of the following: design (risk of bias), consistency across studies, directness of evidence, precision of estimates, and presence of publication bias. We will use GRADEproGDT to create a 'Summary of findings' table to report the quality of the evidence. The GRADE approach yields an assessment of the quality of a body of evidence according to one of four grades.

- High: We are very confident that the true effect lies close to that of the estimate of the effect.

- Moderate: We are moderately confident in the effect estimate: The true effect is likely to be close to the estimate of the effect, but there is a possibility that it is substantially different.

- Low: Our confidence in the effect estimate is limited: The true effect may be substantially different from the estimate of the effect.

- Very low: We have very little confidence in the effect estimate: The true effect is likely to be substantially different from the estimate of effect.

\section{Sensitivity analysis}

We will conduct sensitivity analyses to assess the impact of high risk of bias on the outcome of metaanalyses by adding studies with high risk of bias to pooled studies with low risk of bias.

\section{Discussion}

The intervention essentially works on principle of "Seeing is believing" - behavioral change phenomena. Immediate feedback on quality assurance indicators have shown promising results in various health care set ups and direct visualization of hand hygiene quality through UV fluorescent tracers is one such technique which has gained significant attention during last decade. It basically improves compliance through behavioral change, as actual images of contaminated hands motivate health care workers to adhere to standard hand hygiene protocols.

This innovative imaging tool uses various forms of materials such as powder, gel and lotion. Post hand wash/rub the hands are allowed to be discreetly coated with an invisible layer of UV eliminating material (powder, gel, lotion) which is composed of harmless microscopic particles that simulate microbial contamination and cross-contamination. Afterwards with the use of a UV Forensic Torch or Checkpoint Lamp, contaminated area can be easily differentiated and instant feedback on hand hygiene quality can be obtained by evaluating the missed spots. Real time or immediate feedback employed in this technology are the most efficient tool leading to behavior changes. The visualization of missed areas helps healthcare workers to understand the hand's role in infection transmission. Various studies have clearly showed that imaging based interventions result in effective hand hygiene practices which may have enormous role to play in controlling HAl associated morbidity and mortality [31]. The reduction in 
HAI rate proportionately improves clinical indicators such as number of ICU stays and QALY that may ultimately reduce financial burden associated with the diseases condition.

Translation of results of studies from developed countries on the impact and cost of infections to situations in developing countries may not be appropriate as for developing countries, the spectrum of challenges faced is completely different then the developed part of world. Majority of the Indian public health care facilities needs to deal with the issues related to the patient load, complexity of the disease, availability of the skilled health care providers and affordability [26]. This poses a major problem of demand supply mismatch, not only in the public sector, but also in the private sector since the population that needs to be covered in India is over 1 billion.

It is reported that India has alarmingly high incidence $(\approx 60 \%)$ of HAl especially in ICU than other areas of the hospital and this leads to almost doubling of the treatment cost and prolonged hospital stay [26,30]. This also imparts substantial clinical and economic burden in Indian health care system. The results of this HTA study will help the decision makers for incorporation of such innovative devices in public health system of India for the prevention of HAls.

\section{List Of Abbreviations}

AMR-Antimicrobial Resistance

Cl- Confidence Interval

HAl- Hospital Acquired Infection

HTA- Health Technology Assessment

ICER- Incremental Cost-effectiveness Ratio

ICU- Intensive Care Unit

LMIC- Low and Middle Income Countries

MD- Mean Difference

OD- Odds Difference

OR- Odds Ratio 
QALY- Quality Adjusted Life Years

RCT- Randomized Controlled Trial

UV- Ultraviolate

WHO- World Health Organization

\section{Declarations}

Ethics approval and consent to participate: Not Applicable

Consent for publication: Not Applicable

Availability of data and materials: The datasets used and/or analyzed during the current study are available from the corresponding author on reasonable request.

Competing interest: The authors declare that they have no competing interests

Funding: No internal or external source of support supplied

Authors' contributions: All the three authors prepared and reviewed the protocol and provided suggestions.

Acknowledgements: Not Applicable

Footnotes: Nil

\section{References}

1. Gamalathge PU, Kularatna S, Carter HE, Senanayake S, Graves N. Cost-effectiveness of interventions to reduce the risk of healthcare-acquired infections in middle-income countries: a systematic review. Journal of infection prevention. 2019 Nov;20(6):266-73.

2. Chakravarthy M, Myatra SN, Rosenthal VD, et al. The impact of the International Nosocomial Infection Control Consortium (INICC) multicenter, multidimensional hand hygiene approach in two cities of India. Journal of Infection and Public Health 2015;8:177-86.

3. Clay KA, O'Shea MK, Fletcher T, Moore AJ, Burns DS, Craig D, Adam M, Johnston AM, Bailey MS, Gibson C. Use of an ultraviolet tracer in simulation training for the clinical management of Ebola virus disease. Journal of Hospital Infection. 2015 Nov 1;91(3):275-7.

4. Crook B, Makison Booth C, Hall S. Fluorescence Visualization as a Training Tool for Infection Control. Int J Pub Health Safe. 2018;3(156):2.

5. Fishbein AB, Tellez I, Lin H, Sullivan C, Groll ME. Glow gel hand washing in the waiting room: a novel approach to improving hand hygiene education. Infection control \& hospital epidemiology. 2011 
Jul;32(7):661-6.

6. Hall S, Poller B, Bailey C, Gregory S, Clark R, Roberts P, Tunbridge A, Poran V, Evans C, Crook B. Use of ultraviolet-fluorescence-based simulation in evaluation of personal protective equipment worn for first assessment and care of a patient with suspected high-consequence infectious disease. Journal of Hospital Infection. 2018 Jun 1;99(2):218-28..

7. Hautemanière A, Diguio N, Daval MC, Hunter PR, Hartemann P. Short-term assessment of training of medical students in the use of alcohol-based hand rub using fluorescent-labeled hand rub and skin hydration measurements. American journal of infection control. 2009 May 1;37(4):338-40.

8. Helder OK, Brug J, Looman CW, van Goudoever JB, Kornelisse RF. The impact of an education program on hand hygiene compliance and nosocomial infection incidence in an urban neonatal intensive care unit: an intervention study with before and after comparison. International journal of nursing studies. 2010 Oct 1;47(10):1245-52.

9. Konicki T, Miller E. Use of a simulation intervention to examine differences in nursing students' hand hygiene knowledge, beliefs, and behaviors. Nurse education today. 2016 Oct 1;45:96-101.

10. Lehotsky Á, Szilágyi L, Demeter-Iclănzan A, et al. Education of hand rubbing technique to prospective medical staff, employing UV-based digital imaging technology. Acta Microbiologica et Immunologica Hungarica 2016;63:217-28.

11. Lehotsky A, Szilágyi L, Ferenci T, Kovács L, Pethes R, Wéber G, Haidegger T. Quantitative impact of direct, personal feedback on hand hygiene technique. Journal of Hospital Infection. 2015 Sep 1;91(1):81-4.

12. Macdonald DJ, Mckillop EC, Trotter S, Gray AJ. One plunge or two?-hand disinfection with alcohol gel. International Journal for Quality in Health Care. 2006 Apr 1;18(2):120-2.

13. Macdonald DJ, McKillop EC, Trotter S, Gray AJ. Improving hand-washing performance-a crossover study of hand-washing in the orthopaedic department. The Annals of The Royal College of Surgeons of England. 2006 May;88(3):289-91.

14. Shlomai NO, Rao S, Patole S. Efficacy of interventions to improve hand hygiene compliance in neonatal units: a systematic review and meta-analysis. European Journal of Clinical Microbiology \& Infectious Diseases. 2015 May 1;34(5):887-97..

15. Pan SC, Chen E, Tien KL, Hung IC, Sheng WH, Chen YC, Chang SC. Assessing the thoroughness of hand hygiene:"seeing is believing". American journal of infection control. 2014 Jul 1;42(7):799-801.

16. Poller B, Hall S, Bailey C, Gregory S, Clark R, Roberts P, Tunbridge A, Poran V, Crook B, Evans C. 'VIOLET': a fluorescence-based simulation exercise for training healthcare workers in the use of personal protective equipment. Journal of Hospital Infection. 2018 Jun 1;99(2):229-35.

17. Silva VD, Caetano JÁ, Silva LA, Freitas MM, Almeida PC, Rodrigues JL. Assessment of hand hygiene of nursing and medical students. Revista Da Rede de Enfermagem Do Nordeste 2017;18:257-63.

18. Škodová M, Urra FG, Benítez AG, Romano MR, Ortiz AG. Hand hygiene assessment in the workplace using a UV lamp. American journal of infection control. 2015 Dec 1;43(12):1360-2. 
19. Szilágyi L, Haidegger T, Lehotsky Á, Nagy M, Csonka EA, Sun X, Ooi KL, Fisher D. A large-scale assessment of hand hygiene quality and the effectiveness of the "WHO 6-steps". BMC infectious diseases. $2013 \mathrm{Dec} ; 13(1): 249$.

20. Vanyolos E, Peto K, Viszlai A, Miko I, Furka I, Nemeth N, Orosi P. Usage of ultraviolet test method for monitoring the efficacy of surgical hand rub technique among medical students. Journal of surgical education. 2015 May 1;72(3):530-5.

21. Widmer AF, Conzelmann M, Tomic M, Frei R, Stranden AM. Introducing alcohol-based hand rub for hand hygiene the critical need for training. Infection Control \& Hospital Epidemiology. 2007 Jan;28(1):50-4.

22. Wiles LL, Roberts C, Schmidt K. Keep it clean: a visual approach to reinforce hand hygiene compliance in the emergency department. Journal of Emergency Nursing. 2015 Mar 1;41(2):119-24.

23. Higgins JPT, Thomas J, Chandler J, Cumpston M, Li T, Page MJ, Welch VA (editors). Cochrane Handbook for Systematic Reviews of Interventions version 6.0 (updated July 2019). Cochrane, 2019. Available from www.training.cochrane.org/handbook.

24. The Nordic Cochrane Centre. Review Manager (RevMan). Version 5.3. Copenhagen: The Nordic Cochrane Centre, The Cochrane Collaboration, 2014.

25. Schünemann H, Bro ek J, Guyatt G, Oxman A, editors. Grade Working Group. GRADE Handbook for Grading Quality of Evidence and Strength of Recommendations. www.guidelinedevelopment.org/handbook Updated October 2013.

26. Chacko B, Thomas K, David T, Paul H, Jeyaseelan L, Peter JV. Attributable cost of a nosocomial infection in the intensive care unit: A prospective cohort study. World journal of critical care medicine. 2017 Feb 4;6(1):79.

27. Singh S, Kumar RK, Sundaram KR, Kanjilal B, Nair P. Improving outcomes and reducing costs by modular training in infection control in a resource-limited setting. International Journal for Quality in Health Care. 2012 Dec 1;24(6):641-8.

28. Mythri $\mathrm{H}$, Kashinath KR. Nosocomial infections in patients admitted in intensive care unit of a Tertiary Health Center, India. Annals of medical and health sciences research. 2014;4(5):738-41.

29. De M, Mukherjee D. A Study on Hospital Acquired Infections among Patients in a Tertiary Care Hospital of Darjeeling District, West Bengal. Bengal Journal of Otolaryngology and Head Neck Surgery 2018;26(3):197-206.

30. Choudhuri AH, Chakravarty M, Uppal R. Epidemiology and characteristics of nosocomial infections in critically ill patients in a tertiary care intensive care unit of Northern India. Saudi journal of anaesthesia. 2017 Oct;11(4):402.

31. Storey SJ, FitzGerald G, Moore G, Knights E, Atkinson S, Smith S, Freeman O, Cryer P, Wilson AP. Effect of a contact monitoring system with immediate visual feedback on hand hygiene compliance. Journal of Hospital Infection. 2014 Oct 1;88(2):84-8

\section{Table}


Table 1: Probabilities to be used in the model

Parameters

Probabilities of $\mathrm{HAl}$

Death attributable to $\mathrm{HAl}$

Probabilities of extended ICU stay attributable to HAI

Relative risk of no intervention vs intervention scenario

Cost per patient (HAI) under no intervention

Cost per patient (HAl) with intervention

\section{Sources}

$[28,29]$

[30]

[30]

Meta-analysis (phase 1)

$[26,27\}$

$[27,28]$; Meta-analysis (phase 1)

\section{Figures}

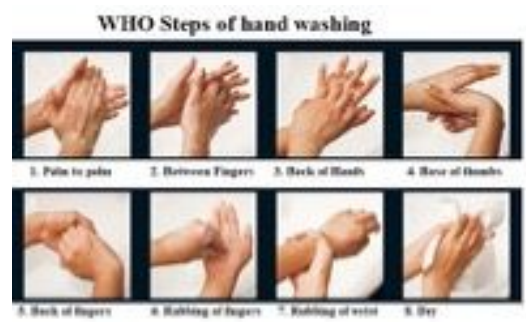

Ianevative UV light based imaging
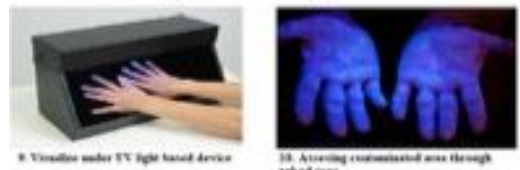

Reference. 1itps//wrux. Who int/gosoclean hasds grotecticnien/

\section{Figure 1}

Description of UV based innovation technique 
-Establish impact of UV light based imaging technology for hand hygiene improvement

- Global sysnthesis evidence analysis)

(Meta-

\section{Phase 1}

Innovation and Hand hygiene

\section{Phase 2}

Hand hygiene \& HAI

-Estimate impact of hand hygiene improvement on HAI from Indian studies

- Use pooled effect obtained from phase 1 to expolate the impact of improve hand hygiene on HAI incidece in India
- Assess costing data (for ICU days \& QALY) of HAI from Indian study

- Asses Cost-effectiveness of the intervention for HAI reduction

\section{Phase 3}

HAI \& Cost-

effectiveness

Figure 2

Overview of the methodology for review 